\title{
Comparison and Fusion of Multiresolution Features for Texture Classification
}

\author{
Shutao $\mathrm{Li}^{1,2}$ and John Shawe-Taylor ${ }^{2}$ \\ ${ }^{1}$ College of Electrical and Information Engineering, Hunan University, Changsha, 410082, P.R. China \\ ${ }^{2}$ Image, Speech and Intelligent Systems Research Group, School of Electronics and Computer Science, University \\ of Southampton, SO17 1BJ, United Kingdom \\ Shutao_li@yahoo.com.cn; jst@ecs.soton.ac.uk
}

\begin{abstract}
In this paper, we investigate the texture classification problem with individual and combined multiresolution features, i.e., dyadic wavelet, wavelet frame, Gabor wavelet, and steerable pyramid. Support vector machines are used as classifiers. The experimental results show that the steerable pyramid and Gabor wavelet classify texture images with the highest accuracy, the wavelet frame follows them, the dyadic wavelet significantly lags behind. Experimental results on fused features demonstrated the combination of two feature sets always outperformed each method individually. And the fused feature sets of multi-orientation decompositions and stationary wavelet achieve the highest accuracy.
\end{abstract}

Keywords: Multiresolution analysis; Texture classification; Support vector machines

\section{Introduction}

In the past few decades, a large number of texture features have been proposed. Tuceryan and Jain (1991) divides these into four major categories, namely, statistical, geometrical, model-based and signal processing features. Recently, one of the major developments in texture classification has been the use of multiresolution and multichannel descriptions. This description provides information about the image contained in time-frequency domain, and thus provides a powerful tool for the description of similar textures. Several multiresolution and multichannel transform algorithms have been used for texture classification, such as the dyadic wavelet transform (Arivazhagan and Ganesan, 2003, Mallat, 1989, Mallat, 1989), wavelet frame transform (Unser, 1995), Gabor filters (Jain and Farrokhnia, 1991, Manjunath and Ma, 1996) and the steerable pyramid (Simoncelli and Freeman, 1995, Karasaridis and Simoncelli, 1996). 
In recent years, the support vector machine (SVM) has emerged as a very successful classification and regression method (Cristianini and Shawe-Taylor, 2000). It has outperformed traditional techniques in various applications such as handwritten digit recognition, text classification, spam categorization and object detection.

In this paper, we investigate the texture classification problem with multiresolution features, i.e., dyadic wavelet, wavelet frame, Gabor wavelet, and steerable pyramid. Support vector machines are used as classifiers. The experimental results show that the steerable pyramid and Gabor wavelet classify the texture images with the highest accuracy, the wavelet frame follows them, the dyadic wavelet significantly lags behind. Experimental results on fused features demonstrated the combination of two feature sets always outperformed each method individually. And the fused feature set of multi-orientation descriptions, i.e., steerable pyramid and Gabor wavelet, and stationary wavelet achieve the highest accuracy.

The rest of this paper is organized as follows. Brief introductions to dyadic wavelet transform, wavelet frame transform, Gabor wavelet and steerable pyramid are given in Section 2. Section 3 describes the feature extraction method and classification results of those four methods and their combinations. The last section gives some concluding remarks.

\section{Multiresolution methods}

\subsection{Dyadic wavelet transform}

The dyadic wavelet transform (DWT) is the most useful technique for multiresolution image analysis (Mallat, 1989, Mallat, 1989). In practice, dyadic wavelet decomposition is carried out using 2 channel filter banks composed of a low-pass (G) and a high-pass (H) filter and each filter bank is then sampled at a half rate (1/2 down sampling) of the previous frequency. By repeating this procedure, it is possible to obtain wavelet transforms of any order. The down-sampling procedure keeps the scaling parameter constant $(n=1 / 2)$ throughout successive wavelet transforms so that it enables a simple computer implementation. In the case of an image, the filtering is implemented in a separable way by filtering the lines and columns. An example is illustrated in Fig.1. The original image through the low-pass filters and high-pass filters can be transformed into four sub-images, namely low-low(LL), low-high(LH), high-low(HL) and high-high(HH).

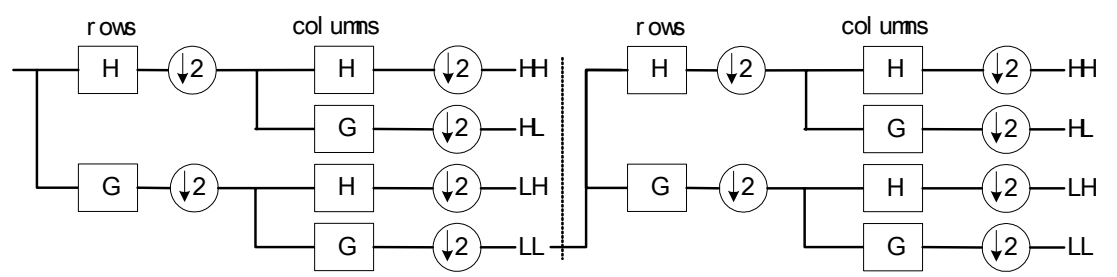

Fig.1. Two-level DWT. 


\subsection{Wavelet frame transform}

The major inconvenience of the dyadic wavelet representation is that it does not conserve an essential property in image processing, which is invariance to translation. Unser (1995) proposed an overcomplete wavelet representation called a wavelet frame transform (WFT). The "overcompleteness" is due to the fact that WFT has no dyadic decimation on each decomposition level, which results in an increase in computational complexity, i.e., both memory requirements and number of flops are $\mathrm{O}(\mathrm{N} \log \mathrm{N})$. Avoiding down-sampling guarantees both aliasing free and translation invariant properties. Although the resulting transform is highly redundant from an information theoretic point of view, it is still simple to compute. A general discrete wavelet frame transform, which corresponds to a two-level decomposition of an image, is presented in Fig.2.

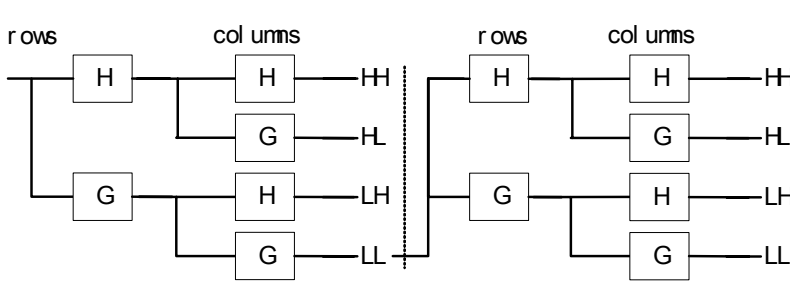

Fig.2. Two-level WFT.

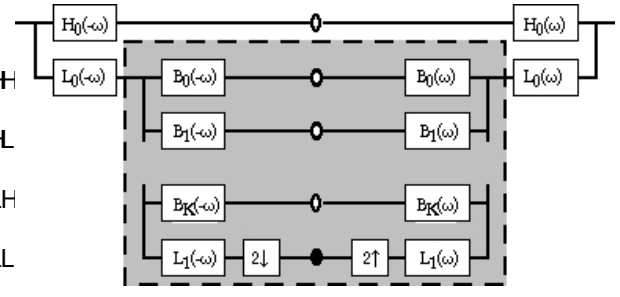

Fig.3. System diagram of SP.

\subsection{Gabor wavelet transform}

A Gabor filter bank is a pseudo-wavelet filter bank where each filter generates a near-independent estimate of the local frequency content. Roughly speaking, a 2-D Gabor filter acts as a local band-pass filter with certain optimal joint localization properties in the spatial domain and in the spatial frequency domain. Given an input image I(x,y), Gabor wavelet transform(GWT) is performed by convolving I(x,y) with a set of Gabor filters of different preferred orientations and spatial frequencies that cover appropriately the spatial frequency domain. Design of a Gabor filter bank for image texture segmentation was proposed by Jain and Farrokhnia (1991). The general functional $\mathrm{g}(\mathrm{x} ; \mathrm{y})$ of the two-dimensional Gabor filter family can be represented as a Gaussian function modulated by an oriented complex sinusoidal signal:

$$
g(x, y)=\frac{1}{2 \pi \sigma_{x} \sigma_{y}} \exp \left[-\frac{1}{2}\left(\frac{\tilde{x}^{2}}{\sigma_{x}^{2}}+\frac{\tilde{y}^{2}}{\sigma_{y}^{2}}\right)+2 \pi j W_{\tilde{x}}\right]
$$

where $\sigma_{x}$ and $\sigma_{y}$ are the scaling parameters of the filter, $\mathrm{W}$ is the center frequency, and $\theta$ determines the orientation of the filter. Gabor filters act as local bandpass filters.

\subsection{Steerable pyramid}

The steerable pyramid (SP) is a linear multi-scale, multi-orientation image decomposition method, unlike most discrete wavelet transforms, which is non-orthogonal and overcomplete (Simoncelli and Freeman, 1995). Fig.3 
shows the analysis/synthesis diagram of steerable pyramid for a single stage. The left-hand side of the diagram is the analysis part and the right hand side is the synthesis part. The circles in between represent the decomposed subband images. The image is first decomposed into lowpass and highpass subbands, using steerable filters $\mathrm{L}_{0}$ and $\mathrm{H}_{0}$. The lowpass band continues to be divided into a set of oriented bandpass subbands $\mathrm{B}_{0}, \ldots, \mathrm{B}_{\mathrm{N}}$ and a lower lowpass subband $\mathrm{L}_{1}$. The lower lowpass subband is subsampled by a factor of 2 along the $\mathrm{x}$ and $\mathrm{y}$ directions. Repeating the shaded area provides the recursive structure.

The steerable pyramid representation is translation-invariant and rotation-invariant. The primary drawback is that the representation is overcomplete by a factor of $4 \mathrm{k} / 3$, where $\mathrm{k}$ is the number of orientation bands.

\section{Test and results}

\subsection{Test dataset}

In this experiment, we use three datasets from two different texture sources: the Brodatz album, the MIT Vision Texture database, which used in most of the experimental settings for texture classification.

The first dataset shown in Fig. 4 has 28 textures, each of size $256 \times 256$. This dataset is challenging because there are significant variations within some textures and some of them are very similar to each other. The second and third datasets both have 10 textures, each of size $128 \times 128$, shown in Fig.5 and Fig.6, respectively. For these two groups, due to the inhomogeneity and large variations, texture types in local windows are perceptually close. All the images have been globally equalized prior to being used.
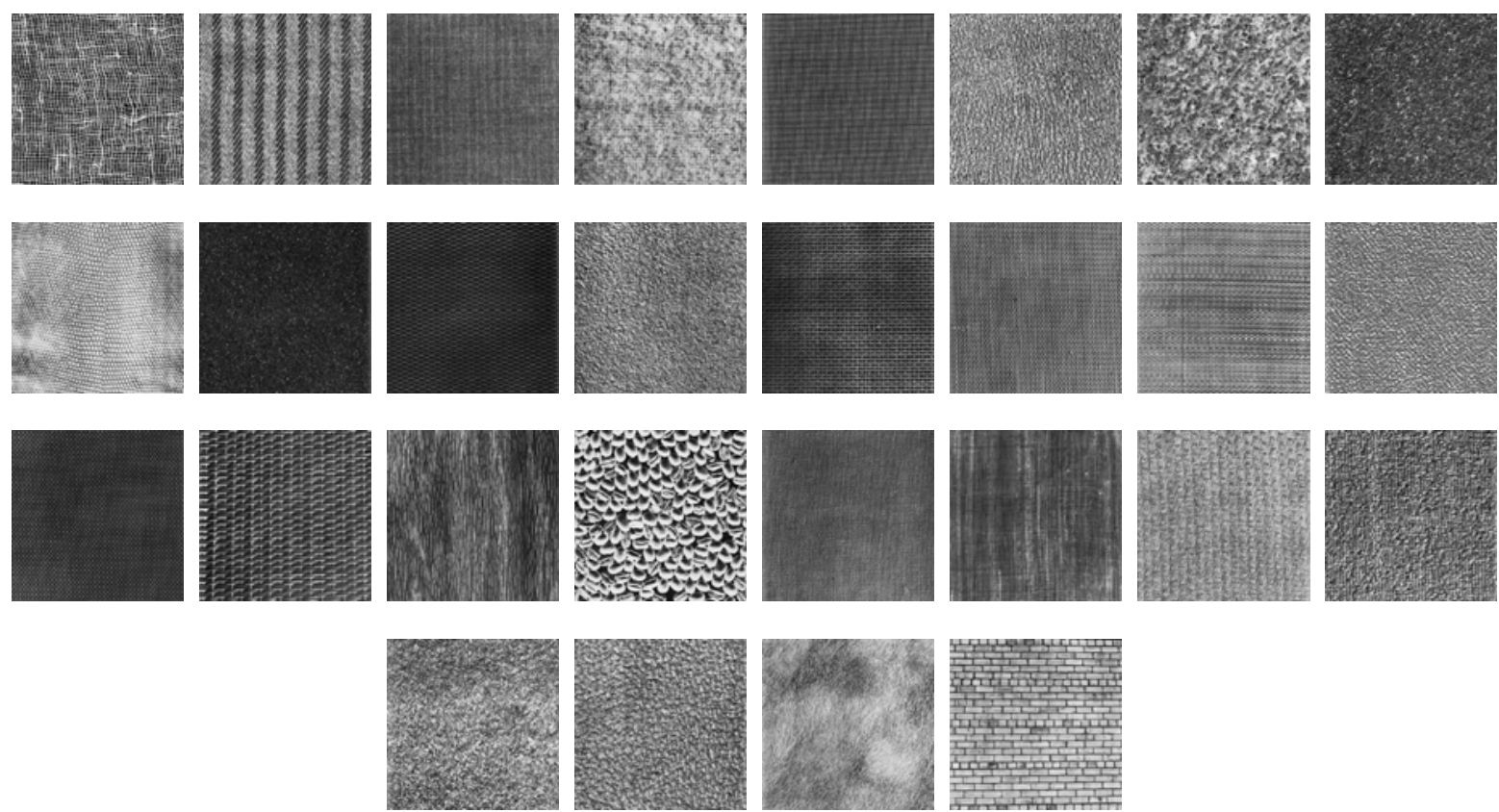

Fig.4. Texture dataset 1 


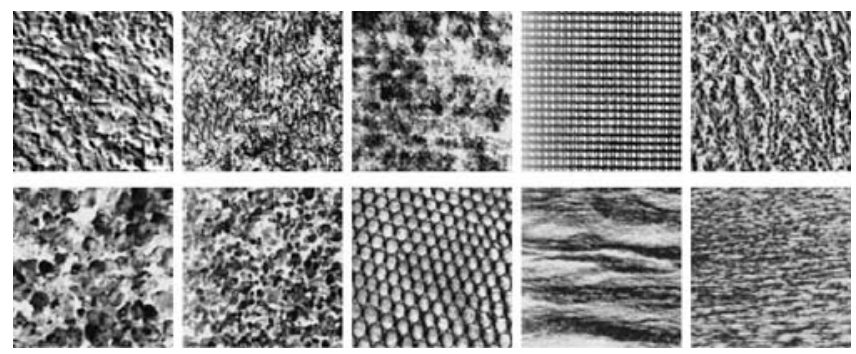

Fig.5. Texture dataset 2

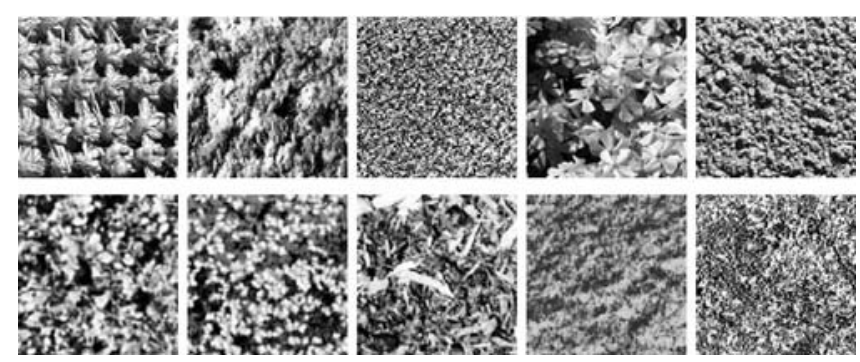

Fig.6. Texture dataset 3

Many previous texture classification studies used overlapping training and test sets, and this is likely to yield unreliable and over-optimistic performance results. Here, we use a complete separation between the training and test sets and repeat the experiment 100 times and compute the average performance.

\subsection{Feature extraction}

Based on common belief, the mean and variance of the energy distribution of the multiresolution transform coefficients for each subband at each decomposition level can be used to identify a texture. Let the image subband be $I_{i}(x, y)$, with $i$ denoting the specific subband, the resulting feature vector $f=\left\{\mu_{i}, \sigma_{i}\right\}$ with,

$$
\begin{aligned}
& \mu_{i}=\frac{1}{M \times N} \sum_{x=1}^{M} \sum_{y=1}^{N}\left|I_{i}(x, y)\right| \\
& \sigma_{i}=\frac{1}{M \times N} \sum_{x=1}^{M} \sum_{y=1}^{N}\left|I_{i}(x, y)-\mu_{n}\right|^{2}
\end{aligned}
$$

where $M$ and $N$ is the size of $I_{i}(x, y)$.

\subsection{SVM setup}

Because the number of texture classes is greater than two, we adopt the conventional one vs. all approach of casting this multi-class classification problem as a number of binary classification problems. In other words, we have one classifier for each texture class, each attempting to separate samples belonging to this class from samples belonging to the other classes. On classifying a new sample, the classifier with the largest output will be selected as the winner, and this new sample is assigned to the winner's corresponding texture class.

In the experiments, the Gaussian kernel will be used in the SVM, because preliminary results suggest that the Gaussian kernel outperforms the polynomial kernel. The gamma value is set to 1 and C value is 1000 . 


\subsection{Experimental results and comparison}

For all the three datasets, the original texture images are subdivided into nonoverlapping samples of size $32 \times 32$. $50 \%$ of the total samples are used for training and the rest are used for testing. The classification results for the four multiresolution decompositions on the three datasets are presented in Table 1. For the dyadic wavelet transform, the texture image regions are decomposed with 3 levels, and db1, db4, db8, coif1, and coif4 basis are used. For the wavelet frame transform, the setup is the same to the dyadic wavelet transform. For the Gabor wavelet transform, the maximum and minimum center frequency are set to 0.4 and 0.05 , the scales and orientations are set to 4 or 6 . For the steerable pyramid, the decomposition filters are sp0filters, sp1filters, sp3filters and sp5filters.

From Table 1, we can see that for the first two datasets, the Gabor wavelet with 6 scales and 6 orientations achieves the highest accuracy. The steerable pyramid with sp5filters follows it. The wavelet frame decompositions with haar basis also have good results. The dyadic wavelet transform is the worst one. For dataset 3, the steerable pyramids with sp1filters and sp3filters have the best classification accuracy. The wavelet frame transform and Gabor wavelet follow it. The dyadic wavelet transform again has the worst performance. From the table, we can say that different wavelet bases result in very different classification performance.

Table 1 Classification results for different individual multiresolution description (\%)

\begin{tabular}{|c|c|c|c|c|}
\hline Multiresolution methods & Number of features & \multicolumn{3}{|c|}{ Test dataset } \\
\cline { 2 - 4 } & & Dataset 1 & Dataset 2 & Dataset 3 \\
\hline DWT(db1,L=3) & 18 & 90.06 & 85.51 & 72.85 \\
\hline DWT(db4, L=3) & 18 & 87.41 & 86.75 & 72.25 \\
\hline DWT(db8, L=3) & 18 & 89.06 & 84.40 & 70.00 \\
\hline DWT(coif1, L=3) & 18 & 89.20 & 88.07 & 72.54 \\
\hline DWT(coif4, L=3) & 18 & $\mathbf{9 3 . 5 8}$ & $\mathbf{8 9 . 1 2}$ & $\mathbf{7 9 . 9 4}$ \\
\hline WFT(db1, L=3) & 18 & $\mathbf{9 6 . 2 0}$ & $\mathbf{9 2 . 4 9}$ & 81.25 \\
\hline WFT(db4, L=3) & 18 & 93.99 & 89.20 & 80.91 \\
\hline WFT(db8, L=3) & 18 & 92.68 & 90.19 & 80.78 \\
\hline WFT(coif1, L=3) & 18 & 94.59 & 90.60 & 81.16 \\
\hline WFT(coif4, L=3) & 18 & 93.34 & 89.77 & 78.15 \\
\hline GWT(s=4,o=4) & 32 & 96.13 & $\mathbf{9 4 . 4 0}$ & 80.94 \\
\hline GWT(s=4,o=6) & 48 & 96.53 & 94.79 & 78.64 \\
\hline GWT(s=6,o=4) & 48 & 95.96 & 93.15 & $\mathbf{8 0 . 2 1}$ \\
\hline GWT(s=6,o=6) & 72 & $\mathbf{9 6 . 5 8}$ & $\mathbf{9 4 . 4 0}$ & 62.88 \\
\hline SP(sp0, L=2) & 6 & 75.44 & 75.21 & $\mathbf{8 6 . 4 9}$ \\
\hline SP(sp1, L=1) & 6 & 87.51 & 89.35 & 85.04 \\
\hline SP(sp3, L=1) & 10 & & 91.16 & \\
\hline
\end{tabular}




\begin{tabular}{|l|l|l|l|l|}
\hline $\mathrm{SP}(\mathrm{sp} 5, \mathrm{~L}=2)$ & 26 & $\mathbf{9 6 . 5 8}$ & $\mathbf{9 2 . 6 3}$ & 82.64 \\
\hline
\end{tabular}

The number of features for all the setups is also shown in Table 1. From the table, we can see that the Gabor wavelet has the largest number of features, compared to the other three methods.

Commonly, different information sources for the same recognition task often lead to different error in the recognition results. So combination of complementary sources will effectively reduce the error rate. Thus, we combine those four different multiresolution decompositions by concatenating them. The classification results of the combined feature sets are shown in Table 2. From the table, it can be concluded that the classification results of combined feature sets are better than those of individual feature sets. And the best accuracies are achieved by the combination of multi-orientation descriptions, i.e. the steerable pyramid and Gabor wavelet, and the wavelet frame transform.

Table 2 Classification results for combined multiresolution descriptions (\%)

\begin{tabular}{|c|c|c|c|c|}
\hline Combination features & \multirow{2}{*}{$\begin{array}{c}\text { Number of } \\
\text { features }\end{array}$} & \multicolumn{3}{|c|}{ Test dataset } \\
\cline { 3 - 5 } & Dataset1 & Dataset2 & Dataset3 \\
\hline DWT(coif4,L=3)+WFT(db1, L=3) & 36 & 96.58 & 92.70 & 81.67 \\
\hline DWT(coif4, L=3)+GWT(s=6,o=6) & 90 & 97.65 & 94.90 & 81.77 \\
\hline DWT(coif4, L=3)+SP(sp5, L=2) & 44 & 98.10 & 94.03 & 84.89 \\
\hline WFT(db1, L=3)+GWT(s=6,o=6) & 90 & 97.47 & $\mathbf{9 5 . 7 6}$ & 81.21 \\
\hline WFT(db1, L=3)+SP(sp5, L=2) & 44 & $\mathbf{9 8 . 4 7}$ & 95.25 & $\mathbf{8 6 . 7 0}$ \\
\hline GWT(s=6,o=6)+SP(sp5, L=2) & 98 & 97.31 & 95.36 & 83.05 \\
\hline
\end{tabular}

\section{Conclusions}

In this paper, we investigated the texture classification problem with multiresolution features, i.e., dyadic wavelet, wavelet frame, Gabor wavelet, and steerable pyramid. Support vector machines are used as classifiers. The experimental results show that the steerable pyramid and Gabor wavelet classify the texture images with the highest accuracy, the wavelet frame follows them, the dyadic wavelet significantly lags behind. Experimental results on fused features demonstrated the combination of two feature sets always outperformed each method individually. Fused feature sets of multi-orientation decompositions, i.e., steerable pyramid and Gabor wavelets, and wavelet frame achieved the highest accuracies.

\section{References}

Arivazhagan, S., Ganesan, L., 2003. Texture Classification using Wavelet Transform. Pattern Recognition Letters 24 (9-10), 1513-1521.

Cristianini, N., Shawe-Taylor, J., 2000. An Introduction to Support Vector Machines. Cambridge University, 
Cambridge, UK.

Jain, A.K., Farrokhnia, F., 1991. Unsupervised Texture Segmentation using Gabor Filters. Pattern Recognition 24 (12), 1167-1186.

Karasaridis, A., Simoncelli, E., 1996. A Filter Design Technique for Steerable Pyramid Image Transforms. In: Proc. Int'l Conf. on Acoust. Speech Signal Process., Atlanta GA, pp.2387-2390.

Manjunath, B. S., Ma, W. Y., 1996. Texture Features for Browsing and Retrieval of Large Image Data. IEEE Trans. Pattern Anal. Machine Intel. 18 (8), 837-842.

Mallat, S.G., 1989. A Theory for Multiresolution Signal Decomposition: The Wavelet Representation. IEEE Trans. Pattern Anal. Machine Intel. 11 (7), 674-693.

Mallat, S.G., 1989. Multifrequency Channel Decompositions of Images and Wavelet Models. IEEE Trans. Acoust. Speech Signal Process., 37 (12), 2091-2110.

Simoncelli, E.P., Freeman, W.T., 1995. The Steerable Pyramid: A Flexible Architecture for Multi-Scale Derivative Computation. In: Proc. IEEE Second Int’l Conf. on Image Process., Washington DC, pp.444-447.

Tuceryan, M., Jain, A.K., 1998. Texture Analysis. In: C.H. Chen, L.F. Pau, and P.S.P. Wang, Ed., Handbook of Pattern Recognition and Computer Vision, 2nd ed., Singapore: World Scientific.

Unser, M., 1995. Texture Classification and Segmentation using Wavelet Frames. IEEE Trans. Image Processing, 4 (11), 1549-1560. 